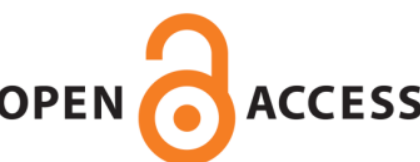

OPEN ACCESS

UWS Academic Portal

\title{
A sphere fitting approach to determine the hip joint centre of the horse
}

Valentin, Stephanie; Peham, Christian; Zsoldos, Rekeba; Licka, Theresia

Published in:

Comparative Exercise Physiology

DOI:

10.3920/CEP160039

E-pub ahead of print: 16/03/2017

Document Version

Peer reviewed version

Link to publication on the UWS Academic Portal

Citation for published version (APA):

Valentin, S., Peham, C., Zsoldos, R., \& Licka, T. (2017). A sphere fitting approach to determine the hip joint centre of the horse. Comparative Exercise Physiology. https://doi.org/10.3920/CEP160039

\section{General rights}

Copyright and moral rights for the publications made accessible in the UWS Academic Portal are retained by the authors and/or other copyright owners and it is a condition of accessing publications that users recognise and abide by the legal requirements associated with these rights. 


\title{
A sphere fitting approach to determine the hip joint centre of the horse
}

S. Valentin ${ }^{\mathrm{a}}$, C. Peham ${ }^{\mathrm{b}}$, R. R. Zsoldos ${ }^{\mathrm{c}}$, T. F. Licka ${ }^{\mathrm{b}, \mathrm{d}}$

${ }^{a}$ Institute for Clinical Exercise \& Health Science, School of Science and Sport, University of the West of Scotland, Hamilton, ML3 OJB, Scotland, United Kingdom

${ }^{\mathrm{b}}$ Equine Clinic, University of Veterinary Medicine, Veterinärplatz 1, 1210 Vienna, Austria

c Working Group Animal Breeding, Department of Sustainable Agricultural Systems, University of Natural Resources and Life Sciences Vienna, Gregor-Mendel-Straße 33, 1180 Vienna, Austria

${ }^{d}$ Large Animal Hospital, Royal (Dick) School of Veterinary Studies, University of Edinburgh, Roslin, EH25 9RG, Scotland, United Kingdom

Corresponding Author:

Stephanie Valentin

stephanie.valentin@uws.ac.uk

Running header: Hip joint centre in the horse

\begin{abstract}
Accurate identification of the hip joint centre (HJC) is crucial for the correct estimation of knee and hip joint loads and kinematics, which is particularly relevant in orthopaedic surgery and musculoskeletal modeling. Several methods have been described for calculation of the HJC in humans, however, no studies have used these methods in the horse despite a similar need for improved evaluation of hip joint biomechanics in rehabilitation and musculoskeletal modeling. This preliminary study uses the commonly used functional method (least-squares sphere fit) to determine the $\mathrm{HJC}$ in three equid cadavers. Bone pins with reflective markers attached were drilled into the tuber coxae (TC), tuber ischium (TI), tuber sacrale (TS), greater trochanter (GT), third trochanter (TT) and lateral femoral condyle (FC) of the uppermost limb of the cadavers positioned in lateral recumbency. Three repetitions of passive movements consisting of pro-and retraction, ab- and adduction and circumduction were performed. The HJC was calculated using a least-squares sphere fitting method and presented as a distance from the TC based on a percentage of the TC to TI vector magnitude. Mean ( \pm standard deviation) of the HJC is located $52.4 \%( \pm 3.9)$ caudally, $0.2 \%( \pm 6.5)$ dorsally, and $19.8 \%( \pm 4.2)$ medially from the TC. This study is the first to quantify the HJC in horses in vitro using a functional method. Further work (in vitro, in vivo and imaging) is required to validate the findings of the present study.
\end{abstract}

\section{Keywords:}

Equine, Ex vivo, Biomechanics, Kinematics 


\section{Introduction}

Identification of the hip joint centre (HJC) is important for the estimation of hip joint kinematics and moments (Cereatti et al., 2009, Ehrig et al., 2006) and for the optimisation of implant placement in hip and knee surgery (Boudroit et al., 2006). Although there is a wealth of literature available on the HJC in man, to our knowledge, the HJC has not been quantified in horses. Identifying the $\mathrm{HJC}$ in horses is mandatory for musculoskeletal modelling purposes. In addition, it can improve the evaluation of pelvic limb kinematics in pathology such as hip (sub)luxation (Barr and Fairburn, 2014) and osteoarthritis (Lamb and Morris, 1987).

Functional and predictive methods have been used to identify the HJC in man (Camomilla et al., 2006). Predictive methods are based on regression equations and anthropometric measurements (Fiorentino et al., 2015) or medical imaging (De Momi et al., 2009). The functional method assumes a spherical ball and socket joint with the geometric centre of the acetabulum representing the centre of rotation of the hip joint; this is quantified by tracking movement of the femur relative to the pelvis (Camomilla et al., 2006). Several algorithms based on sphere-fitting have been used for the functional approach in man (e.g. Gamage and Lasenby, 2002, Piazza et al., 2001), with good accuracy and repeatability (Camomilla et al., 2006; Ehrig et al., 2006).

Although the functional method is more accurate than the predictive method (Fiorentino et al., 2015, Hicks and Richards, 2005), it is nonetheless prone to errors from skin and soft tissue displacement when skin markers are used (Cereatti et al., 2009). While skin displacement of $5 \mathrm{~mm}$ over the greater trochanter in the direction perpendicular to the long axis of the femur has been reported in man during walking (Leardini et al., 2005), in horses over the same location and direction, skin displacement of 142mm has been identified during trot (van Weeren et al., 1990). This could make the use of skin markers less reliable in horses, suggesting the need for bone markers for HJC calculation in the first instance. Subject-specific data obtained from medical imaging have improved HJC accuracy in man (Fiorentino et al., 2015) although this approach can increase exposure to ionising radiation and has longer post-processing time and associated costs (Kainz et al., 2015). Magnetic resonance imaging or computed tomography of the horse hip joint in vivo is limited due to the bore diameter of closed MRI and CT systems. Therefore, the aim of this preliminary study is to identify the equine HJC using bone pins in cadavers using a sphere-fitting method.

\section{Methods}

Three fresh equine cadavers were used in the study (3-year old $495 \mathrm{~kg}$ Standardbred Trotter gelding, 23-year old $583 \mathrm{~kg}$ Holsteiner gelding, 7-year old $382 \mathrm{~kg}$ Appaloosa gelding). These horses were euthanized based on clinical findings unrelated to the study, and on admission to the clinic owners had given permission for the use of the bodies. Cadaver preparation took place immediately after euthanasia and data collection was completed prior to rigor mortis onset. Two horses were placed in right lateral recumbency and one in left lateral recumbency (dependent on their position at euthanasia). Drill bits $(5 \mathrm{~mm}$ diameter) were drilled into the upper most pelvic limb at the Tuber Coxae (TC), Tuber Sacrale (TS), Tuber Ischium (TI), cranial part of the Greater Trochanter (GT), Third Trochanter (TT), and the lateral Femoral Condyle (FC). Reflective markers (15mm diameter) were attached to each of the bone pins (Figure 1). The pelvis was not fixed for logistical reasons.

Figure 1 - (a) Locations of the Tuber Coxae (TC), Tuber Ischium (TI), cranial part of the Greater Trochanter (GT), Third Trochanter (TT), and lateral Femoral Condyle (FC) on horse 1. Note that the Tuber Sacrale (TS) is not visible in this image (b) Isolated pelvis and femur 
bone specimen from an unrelated horse, placed in a similar orientation as the horse in image 1a. The $x, y, z$ coordinate system is displayed.

Kinematic data were collected using eight infrared cameras (Eagle Digital Real Time System, Motion Analysis Corp., USA) and kinematic software (Cortex 1.3) sampling at $120 \mathrm{~Hz}$ whilst an experienced equine orthopaedic surgeon manually moved the limb through three cycles of pro-and retraction, ab-and adduction and circumduction (cranial-lateral-caudal-medial-cranial) of the uppermost pelvic limb. Accuracy of the functional method does not rely on repeated motion patterns being highly comparable, although similar movement patterns were produced from visual inspection of the video and kinematic data.

Kinematic data were processed and smoothed using a $6 \mathrm{~Hz}$ low pass Butterworth filter. A local coordinate system was defined in the pelvis using the pelvic markers. The $\mathrm{x}$-axis was defined by the TC and TI, the y-axis by the TC and TS, and the cross-product calculated to obtain the normalised z-axis. Therefore the calculated z-axis was orthogonal to the determined $\mathrm{x}-\mathrm{y}$ plane. Then the cross product of the defined $x$-axis and calculated z-axis was calculated to obtain the normalised y-axis. As such, the calculated $y$-axis was orthogonal to the $\mathrm{x}-\mathrm{z}$ plane. Therefore the local coordinate system was composed of defined $x$-axis and calculated $y$ - and z-axes. The femoral markers were defined in the local pelvic coordinate system. A least squares method adapted from Jennings (2013) was used to calculate the coordinates of the HJC in the pelvic coordinate system for each of the femoral markers individually, and then the mean HJC location was calculated. The HJC was presented as a percentage of the TC-TI vector magnitude in each of the three axes from the TC.

\section{Results}

All movement trials could be used. There was no noticeable trend for increased or decreased movement over the three measurements, indicating that the soft tissues had not loosened or that rigor mortis had occurred during the measurements. Range of craniocaudal displacement (along the $\mathrm{x}$-axis) of FC in the pelvic coordinate system was 105.7 - $316.0 \mathrm{~mm}$ across all horses, movement directions and trials. For lateromedial displacement (z-axis), these values were 152.7 - $275.8 \mathrm{~mm}$ respectively (Figure 2).

Figure 2 - (a) Craniocaudal range of motion ( $\mathrm{mm}$ ) of the lateral femoral condyle marker in the pelvic coordinate system of each horse and movement trial (b) Lateromedial range of motion $(\mathrm{mm})$ of the lateral femoral condyle marker in the pelvic coordinate system of each horse and movement trial.

Vector magnitude of TC-TI ranged from 515.6-597.9 mm across all horses. The mean ( \pm standard deviation) HJC was located $52.4 \%( \pm 3.9)$ caudally, $0.2 \%( \pm 6.5)$ dorsally, and $19.8 \%$ $( \pm 4.2)$ medially from the TC, based on TC-TI vector magnitude (Figure 3 ).

Figure 3 - Location of the Hip Joint Centre for each of the femoral markers individually and the average location, presented as a distance from the tuber coxae in the (a) craniocaudal direction (b) dorsoventral direction and (c) mediolateral direction. Values are reported as a percentage $(\%)$ of the tuber coxae to tuber ischium vector magnitude. Error bars indicate the standard deviations. 


\section{Discussion}

This preliminary study reports on the equine HJC, determined using a functional method and bone-fixated markers in vitro. The gold standard for HJC quantification in humans is by medical imaging (Kainz et al., 2015). Unfortunately, similar data are not available in the horse therefore the results from the present study cannot be directly compared. However, as the functional method has successfully been used to determine the HJC in man in vitro (Cereatti et al., 2009), it is anticipated that the similar approach used in the present study has provided a good initial estimation of the equine HJC.

In the present study, a variety of body types were used (Warmblood, Standardbred Trotter, and Appaloosa) to illustrate the universal application of the proposed HJC. In future studies, ponies and cold blood horses should also be used to quantify the HJC in a more generalised population. Furthermore, data from greater sample sizes of specimens from different breeds or types could provide breed-specific HJC locations for even greater accuracy. This is particularly relevant in light of conformation differences amongst different breeds of horses, e.g. the slope of the pelvis can vary significantly amongst groups/types of elite performance horses (Holmström et al., 1990).

The disadvantage of using fresh specimens with all soft tissue remaining in situ is that soft tissue changes occur with time after death. However, changes in range of craniocaudal and lateromedial displacement of the distal femur between first and last passive movements performed in the present study were minimal and did not display an increasing or decreasing pattern, therefore it is unlikely that tissue changes influenced the study findings. Drilling through the muscle was chosen over dissection of the limb down to only the joint capsule and its ligaments to retain a more natural range of motion, as step-wise reduction of soft tissues has been shown to reduce joint stiffness (Valentin et al., 2012).

In the present study we used individual markers drilled into bony landmarks of the pelvis and proximal and distal femur. Although previous work has reported that skin-fixated distal marker clusters as less prone to soft tissue artefacts (Cereatti et al., 2009) and therefore probably more reliable, the most proximal femoral marker (GT) in the present study had the smallest standard deviation for two of the three planes. This illustrates the advantage of using bone pins rather than skin fixated markers. Although marker clusters are recommended over individual markers placed over bony landmarks when non-sagittal plane movements are performed (Besier et al., 2003; Borhani et al., 2013), sagittal plane movement is the primary movement available in the equine hip, therefore the use of individual markers should not have greatly influenced the study results.

In humans, the femoral head is described as choncoid rather than strictly spherical (Menschik, 1997) which may affect movement of the femoral head in the acetabulum and thereby influence the HJC. However, translations of the HJC were of the same magnitude in cadavers using bone pins as that of a mechanical analogue modelled using a spherical hinge (Cereatti et al., 2010). Although the femoral head of the horse is commonly described as semi-spherical (Budras et al., 2001), it shows less convexity in its cranial half than in its caudal half (Figure 4). As the horse has a relatively large fovea, a strong ligamentum capitis femoris, an accessory ligament and the labrum supplementing the socket shape of the acetabulum, only a small degree of translation is anticipated in this joint. Nonetheless it is recommended that a study similar to Cereatti and colleagues (2010) is performed in the horse to determine the influence of femoral head shape and translation on HJC location. 
Figure 4 - Non-spherical head of the femur (less convexity in the cranial half than the caudal half)

A potential source of error in $\mathrm{HJC}$ estimation when using the functional method is displacement of the pelvis when the femur is moved. The pelvis was not fixed to the supporting surface in the present study for logistical reasons, although not deemed necessary either, as the mass of the cadaver was judged to be sufficient to minimise gross pelvic displacement. Furthermore, it is suggested that a least squares approach such as that used in the present study can minimise the error source caused by a mobile pelvis (Piazza et al., 2004). An alternative approach would be to use a Monte Carlo simulation as described by De Momi et al (2009), which uses an initial estimation of the HJC described by Siston and Delp (2006). This has shown good reliability and accuracy regardless of large pelvic displacements, therefore this method might be considered for future experiments investigating the HJC in horses.

Limited range of hip joint motion can be a possible error source in HJC estimation when using the functional method (Piazza et al., 2001; Piazza et al., 2004). Although the range of flexionextension in the equine hip joint is large, movement in other directions is limited (Dyce et al., 2010). The International Society of Biomechanics (ISB) recommends the functional method for the identification of the $\mathrm{HJC}$ in people with adequate hip range of motion, and the predictive method in populations with limited hip range of motion (Wu et al., 2002). As the functional method is more accurate than the predictive method (Fiorentino et al., 2015, Sangeux et al., 2011), the functional method was used in the present study however future work should also quantify the equine $\mathrm{HJC}$ using the predictive method. This will determine which method of HJC estimation is most robust in the horse.

Although further work is required to establish the reliability of the findings reported in the present study, this preliminary work is an important and necessary step for the development of musculoskeletal models of the equine pelvis. In the last decade, musculoskeletal modeling of the equine forelimb has made considerable advancements (Harrison et al., 2012, Swanstrom et al., 2005, Zarucco et al., 2006). This is in contrast to the equine hindlimb, likely due to the complex function of the pelvic girdle. It is anticipated that the results presented here can assist in the development of musculoskeletal models of the equine pelvic limb.

In conclusion, the present study provides a preliminary estimation of the HJC in the horse using a functional method. Future HJC studies in horses should use other methods commonly applied in humans including the predictive method and imaging, such that comparisons can be made to evaluate the reliability of the results reported.

\section{Acknowledgements}

No funding was received in support of this study

\section{Conflict of Interest}

None of the authors have a conflict of interest to declare.

\section{References}

Barr, A.R.S., Fairburn, A.J., 2014. Aspects of coxofemoral luxations in the horse. Equine Veterinary Education 26: 259-261.

Besier, T.F., Sturnieks, D.L., Alderson, J.A., Lloyd, D.G., 2003. Repeatability of gait data using a functional hip joint centre and a mean helical knee axis. Journal of Biomechanics 36 : 1159-1168.

Borhani, M., McGregor, A.H., Bull, A.M., 2013. An alternative technical marker set for the pelvis is more repeatable than the standard pelvic marker set. Gait and Posture 38: 1032-1037. 
Boudroit, U., Hilgert, J., Hinrichs, F., 2006. Determination of the rotational center of the hip. Archives of Orthopaedic and Trauma Surgery 126: 417-420.

Budras, K.D., Sack, W.O., Roeck, S., 2001. Anatomy of the horse - An illustrated text. Schluetersche GmBH \& Co, Hannover, Germany.

Camomilla, V., Cereatti, A., Vannozzi, G., Cappozzo, A., 2006. An optimized protocol for hip joint centre determination using the functional method. Journal of Biomechanics 39: 10961106.

Cereatti, A., Donati, M., Camomilla, V., Margheritini, F., Cappozzo, A., 2009. Hip joint centre location: an ex vivo study. Journal of Biomechanics 42: 818-823.

Cereatti, A., Marghertini, F., Donati, M., Capozzo, A., 2010. Is the human acetabulofemoral joint spherical? Journal of Bone and Joint Surgery 92-B: 311-14.

De Momi, E., Lopomo, N., Cerveri, P., Zaffagnini, S., Safram, MR., Ferrigno, G., 2009. Invitro experimental assessment of a new robust algorithm for hip joint centre estimation. Journal of Biomechanics 42: 989-995.

Dyce, K.M., Sack, W.O., Wensing, C.J.G., 2010. The Locomotor Apparatus. In: Textbook of Veterinary Anatomy. Saunders Elsevier, Missouri.

Ehrig, R.M., Taylor, W.R., Duda, G.N., Heller, M.O., 2006. A survey of formal methods for determining the centre of rotation of ball joints. Journal of Biomechanics 39: 2798-2809.

Fiorentino, N.M., Kutschke, M.J., Atkins, P.R., Foreman, K.B., Kapron, A.L., Anderson A.E., 2015. Accuracy of functional and predictive methods to calculate the hip joint center in young non-pathologic asymptomatic adults with dual fluoroscopy as a reference standard. Annals of Biomedical Engineering 44: 2168-80.

Gamage, S.S., Lasenby, J., 2002. New least squares solutions for estimating the average centre of rotation and the axis of rotation. Journal of Biomechanics 35: 87-93.

Harrison, S.M., Whitton, R.C., King, M., Haussler, K.K., Kawcak, C.E., Stover, S.M., Pandy, M.G., 2012. Forelimb muscle activity during equine locomotion. Journal of Experimental Biology 215: 2980-2991.

Hicks, J.L., Richards, J.G., 2005. Clinical applicability of using spherical fitting to find hip joint centers. Gait and Posture 22: 138-145.

Holmström, M., Magnusson, L.E., Philipsson, J., 1990. Variation in conformation of Swedish Warmblood horses and conformational characterisitic of élite sport horses. Equine Veterinary Journal 22: 186-193.

Jennings (2013) http://www.mathworks.com/matlabcentral/fileexchange/34129-sphere-fit-least-squared-/content/sphereFit/sphereFit.m

Kainz, H., Carty, C.P., Modenese, L., Boydd, R.N., Lloyd, D.G., 2015. Estimation of the hip joint centre in human motion analysis: A systematic review. Clinical Biomechanics 30: 319329.

Lamb, C.R., Morris, E.A., 1987. Coxofemoral arthrosis in an aged mare. Equine Veterinary Journal 19: 350-352.

Leardini, A., Chiari L., Croce, U.G., Cappozzo A., 2005. Human movement analysis using stereophotogrammetry. Part 3. Soft tissue artifact assessment and compensation. Gait and Posture 21: 212-225.

Menschik, F., 1997. The hip joint as a conchoid shape. Journal of Biomechanics 30: 971-3. 
Piazza, S.J., Okita, N., Cavanagh, P.R., 2001. Accuracy of the functional method of hip joint center location: effects of limited motion and varied implementation. Journal of Biomechanics 34: 967-973.

Piazza, S.J., Erdemir, A., Okita, N., Cavanagh, P.R., 2004. Assessment of the functional method of hip joint center location subject to reduced range of hip motion. Journal of Biomechanics 37: 349-356.

Sangeux, M., Peters, A., Baker, R., 2011. Hip joint centre localization: evaluation on normal subjects in the context of gait analysis. Gait and Posture 34: 324-328:

Siston, R.A., Delp, S.L., 2006. Evaluation of a new algorithm to determine the hip joint center. Journal of Biomechanics 39: 125-130.

Swanstrom, M.D., Zarucco, L., Hubbard, M., Stover, S.M., Hawkins, D.A., 2005. Musculoskeletal modeling and dynamic simulation of the thoroughbred equine forelimb during stance phase of the gallop. Journal of biomechanical engineering 127: 318-328.

van Weeren, P.R., van den Bogert, A.J., Barneveld, A., 1990. A quantitative analysis of skin displacement in the trotting horse. Equine Veterinary Journal 22: 101-109.

Valentin, S., Groesel, M., Licka, T., 2012. The presence of long spinal muscles increases stiffness and hysteresis of the caprine spine in-vitro. Journal of Biomechanics 45: 2506-2512.

Wu, G., Siegler, S., Allard, P., Kirtley, C., Leardini, A., Rosenbaum, D., Whittle, M., D'Lima,D.D., Cristofolini, L., Witte, H., Schmid, O., Stokes, I., 2002. ISB recommendation on definitions of joint coordinate system of various joints for the reporting of human joint motionpart I: ankle, hip, and spine. International Society of Biomechanics. Journal of Biomechanics 35: 543-548.

Zarucco, L., Wisner, E.R., Swanstrom, M.D., Stover, S.M. 2006. Image fusion of computed tomographic and magnetic resonance images for the development of a three-dimensional musculoskeletal model of the equine forelimb. Veterinary Radiology \& Ultrasound 47: 553562. 
Fig1

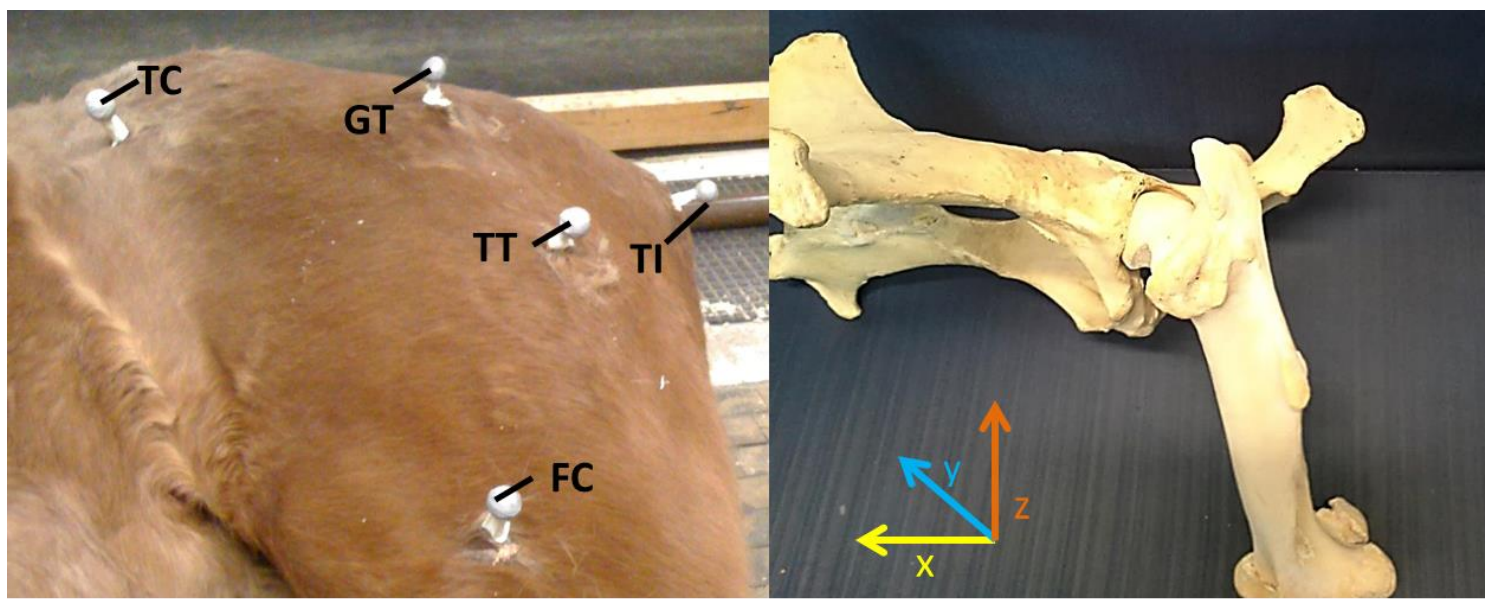

Fig2

a

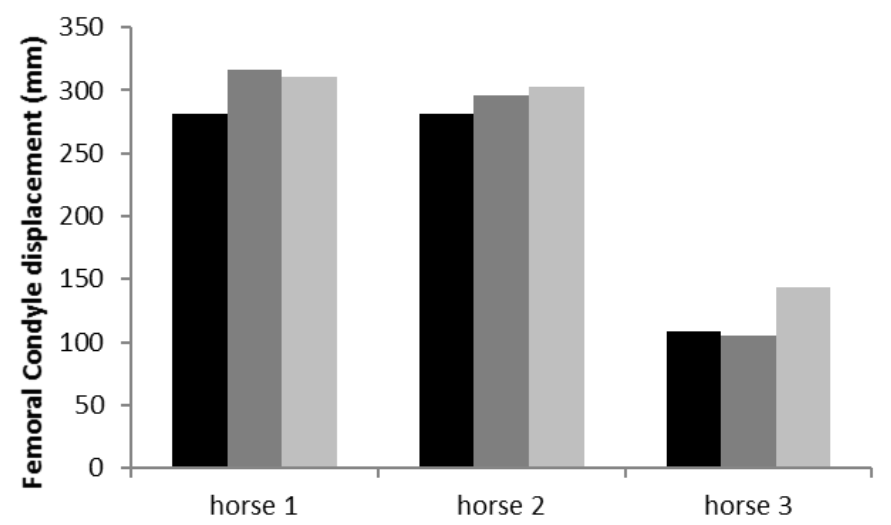

- trial 1

- trial 2

trial 3

b

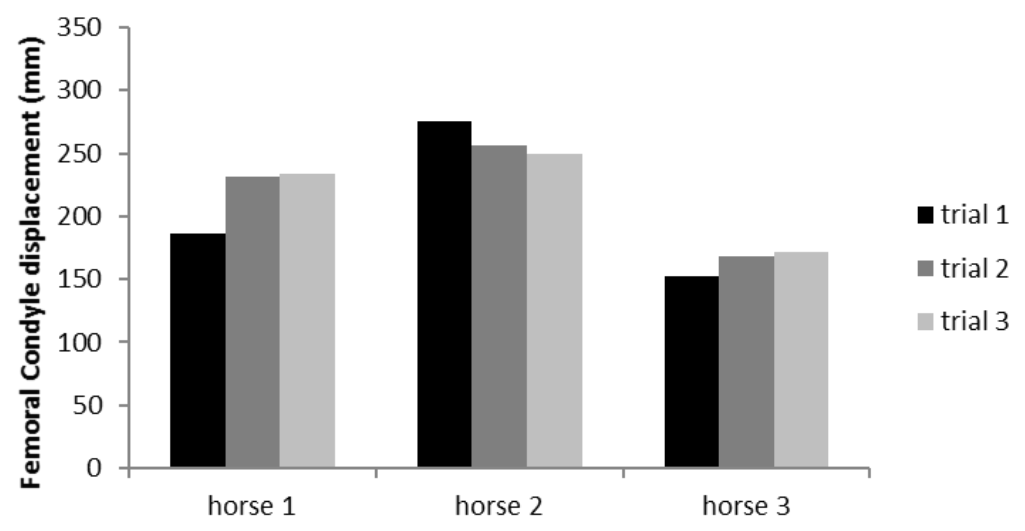


Fig3
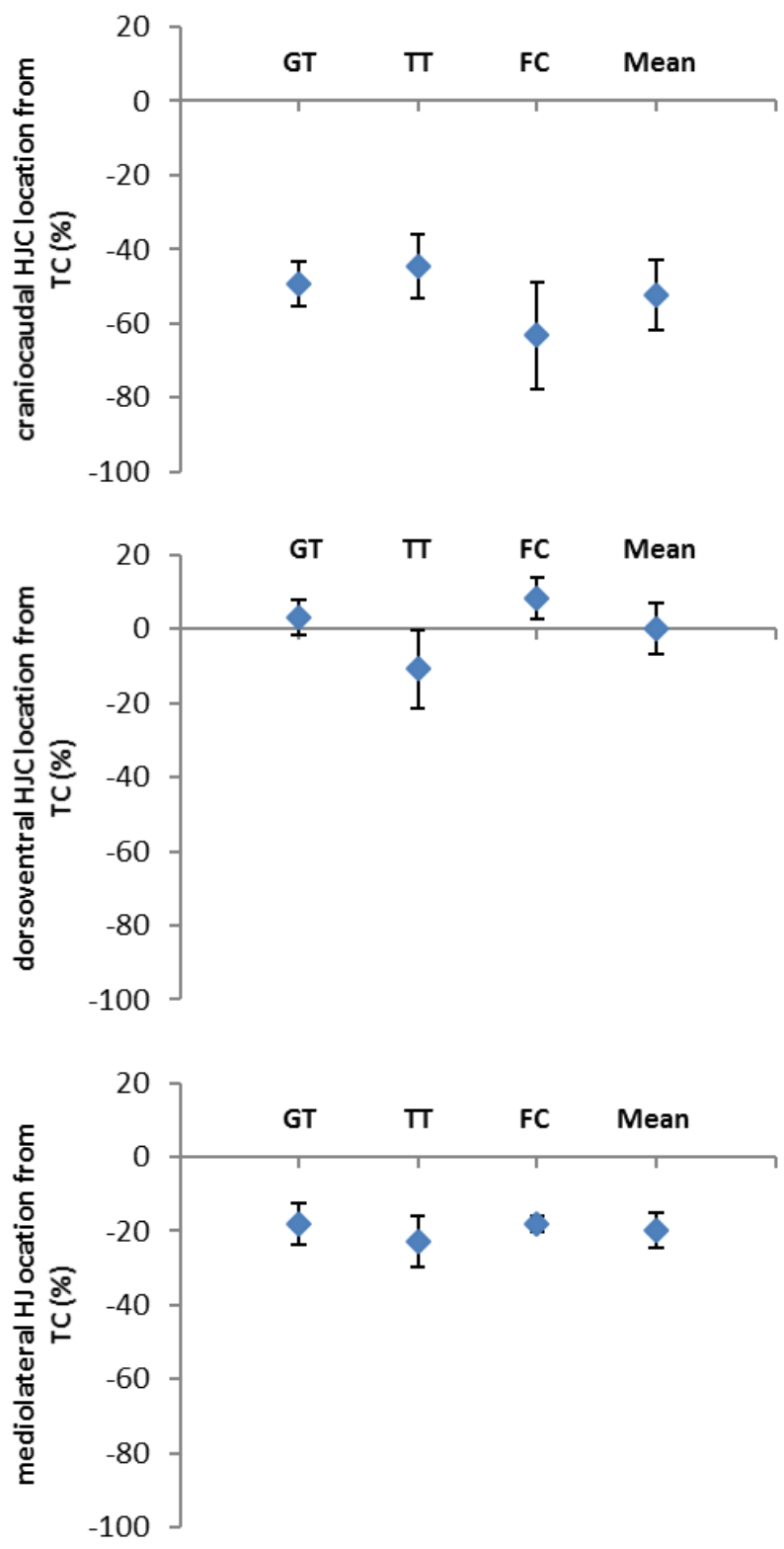

Fig4

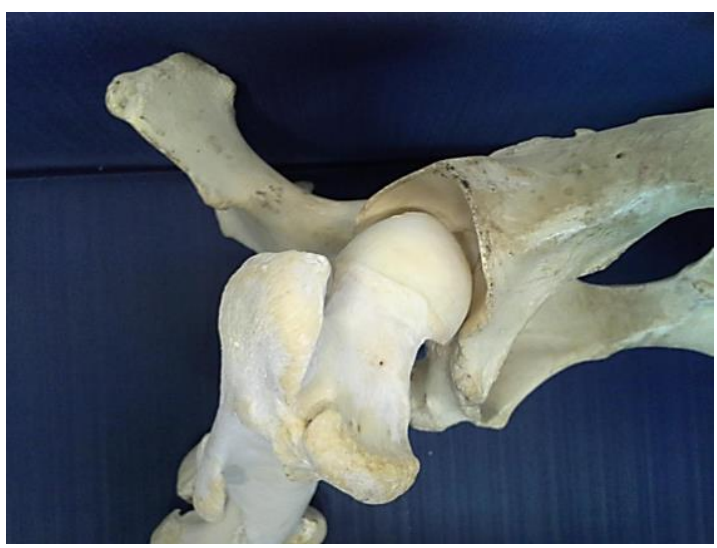

